most susceptible to HIV, this could mean that infants are less prone to the infection spreading. Moreover, babies are born without central memory $\mathrm{T}$ cells, so they are likely to have a smaller reservoir of infected cells, says Mike McCune, an immunologist at the University of California, San Francisco.

The IMPAACT study, to be conducted across some of the group's 71 sites worldwide, will screen and treat hundreds of babies to find 20-30 infants who have acquired HIV from untreated mothers or from those whose HIV was not well controlled during pregnancy. Because diagnosing HIV takes up to 7 days, all screened babies will automatically receive a similar treatment to the Mississippi baby: a cocktail of three drugs within 48 hours of birth. Physicians will add a fourth drug if babies then test positive for HIV. Around the age of three, the 20-30 children will be tested to see whether their immune systems make antibodies to HIV or if it can be detected in their blood. Those testing negative on both counts would then be taken off the drugs to see whether they can remain HIV-free.

The practical and ethical challenges of the trial are significant. Babies of untreated HIVpositive women have only a $15-30 \%$ chance of infection at birth, so the trial will need to recruit many babies to try to cure the few who are infected. Those who do not contract HIV will be treated anyway, perhaps exposing them to drug side effects. These are usually mild, but can deplete certain blood cells.

But children born to untreated HIVpositive women are already given up to three drugs after birth as a precaution. The potential for finding a cure far outweighs the risks of adding another drug, or of stopping treatment to test whether the cure has worked, says Yvonne Bryson, a physician at the Mattel Children's Hospital at the University of California, Los Angeles, and co-chair of the trial. "There's much more benefit to be gained than risk," she says.

Physicians are already considering changing the way they treat children infected by the virus. Bryson says that families of HIVpositive teenagers who were treated soon after birth and kept on medication are now asking that the teens be taken off the drugs.

Ultimately, the 34 million people worldwide who live with HIV could also benefit, researchers say. If it turns out that infants are more amenable to cures because they have a less active inflammatory response, that might encourage physicians to prescribe treatments that are less likely to trigger inflammation in adults, McCune says.

Bryson, who has worked on HIV since the first case was detected, thinks that the end could be in sight. "I saw patient zero," Bryson says. "I've always been so excited to think that I would see the day that we would arrive at a cure, and I think we're here." -

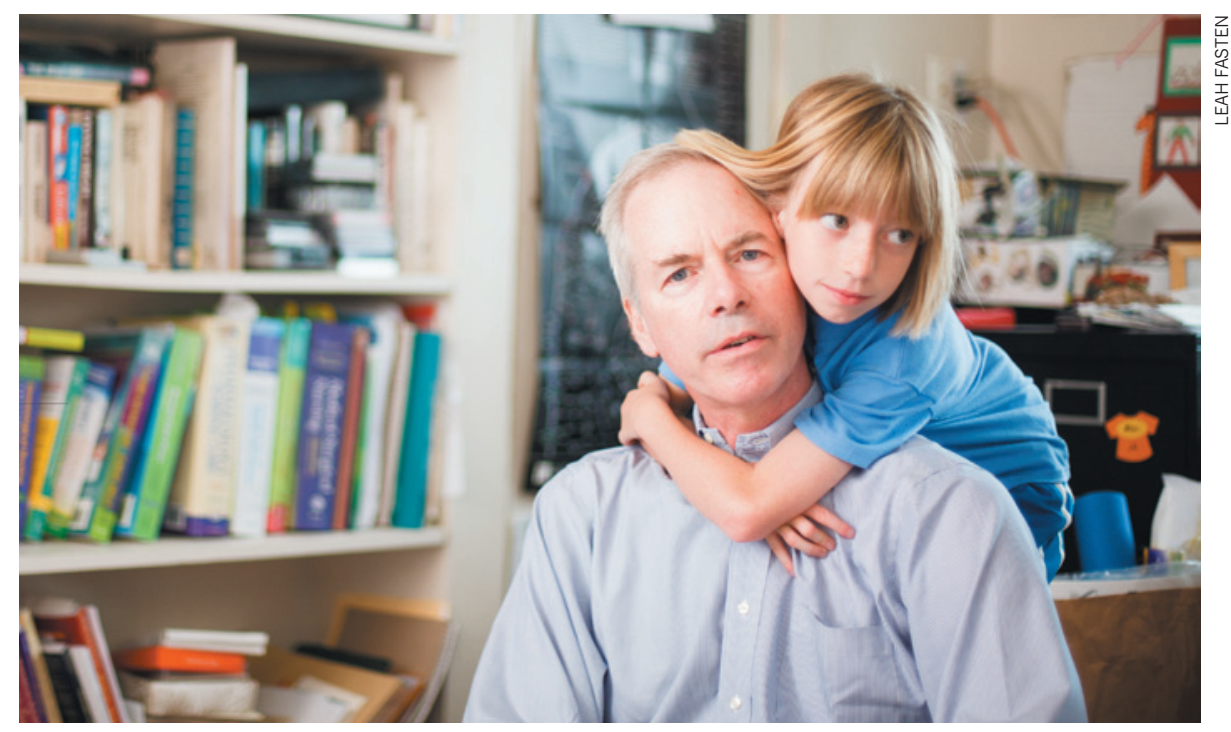

Hugh Rienhoff prepared his daughter's DNA for sequencing at home using second-hand equipment.

\title{
PERSONAL GENOMICS
}

\section{Father's genetic quest pays off}

\section{Mutation provides clue to daughter's undefined syndrome.}

\section{BY BRENDAN MAHER}

$\mathrm{H}$ ugh Rienhoff says that his nine-yearold daughter, Bea, is "a fire cracker", "a tomboy" and "a very sassy, impudent girl”. But in a forthcoming research paper, he uses rather different terms, describing her hypertelorism (wide spacing between the eyes) and bifid uvula (a cleft in the tissue that hangs from the back of the palate). Both are probably features of a genetic syndrome that Rienhoff has obsessed over since soon after Bea's birth in 2003. Unable to put on much muscle mass, Bea wears braces on her skinny legs to steady her on her curled feet. She is otherwise healthy, but Rienhoff has long worried that his daughter's condition might come with serious heart problems.

Rienhoff, a biotech entrepreneur in San Carlos, California, who had trained as a clinical geneticist in the 1980s, went from doctor to doctor looking for a diagnosis. He bought lab equipment so that he could study his daughter's DNA himself - and in the process, he became a symbol for the do-it-yourself biology movement, and a trailblazer in using DNA technologies to diagnose a rare disease (see Nature 449, 773-776; 2007).

"Talk about personal genomics," says Gary Schroth, a research and development director at the genome-sequencing company

Illumina in San Diego, California, who has helped Rienhoff in his search for clues. "It doesn't get any more personal than trying to figure out what's wrong with your own kid."

Now nearly a decade into his quest, Rienhoff has arrived at an answer. Through the partial-genome sequencing of his entire family, he and a group of collaborators have found a mutation in the gene that encodes transforming growth factor- $\beta 3$ (TGF- $\beta 3$ ). Genes in the TGF- $\beta$ pathway control embryogenesis, cell differentiation and cell death, and mutations in several related genes have been associated with Marfan syndrome and Loeys-Dietz syndrome, both of which have symptomatic overlap with Bea's condition. The mutation, which has not been connected to any disease before, seems to be responsible for Bea's clinical features, according to a paper to be published in the American Journal of Medical Genetics.

Hal Dietz, a clinician at Johns Hopkins University School of Medicine in Baltimore, Maryland, where Rienhoff trained as a geneticist, isn't surprised that the genetic culprit is in

ONATURE.COM For more on Hugh Rienhoff's genetic search, see: go.nature.com/quvtks this pathway. "The overwhelming early hypothesis was that this was related," says Dietz, who co-discovered LoeysDietz syndrome in 2005. 
Rienhoff had long been tapping experts such as Dietz for assistance. In 2005, an examination at Johns Hopkins revealed Bea's bifid uvula. This feature, combined with others, suggested Loeys-Dietz syndrome, which is caused by mutations in TGF- $\beta$ receptors. But physicians found none of the known mutations after sequencing these genes individually. This was a relief: Loeys-Dietz is associated with devastating cardiovascular complications and an average life span of 26 years.

In 2008, Jay Flatley, chief executive of Illumina, offered Rienhoff the chance to sequence Bea's transcriptome - all of the RNA expressed by a sample of her cells along with those of her parents and her two brothers. After drilling into the data, Rienhoff and his collaborators found that Bea had inherited from each parent a defectivelooking copy of CPNE1, a poorly studied gene that seems to encode a membrane protein. It looked like the answer.

But questions remained. The gene did not have obvious connections to Bea's features, and publicly available genome data suggests that the CPNE1 mutations are present in about 1 in 1,000 people - an indication that there should be many more people like Bea.

Unsatisfied, Rienhoff went back to Illumina in 2009 to ask for more help. He proposed exome sequencing, which captures the whole protein-encoding portion of the genome, and is in some ways more comprehensive than transcriptome sequencing. At the time, Illumina was developing its exome-sequencing technology, and the company again took on the Rienhoff family as a test group.

The analysis pulled up a mutation in one copy of the gene that encodes TGF- $\beta 3$ — just in Bea. In cell culture and experiments in frog eggs, the faulty gene seems to produce a non-functional protein that reduces TGF- $\beta$ signalling. This mechanism would differ from what many suspect is going on in Marfan and Loeys-Dietz syndromes, in which mutations paradoxically amp up TGF- $\beta$ signalling. A collaborator of Rienhoff is now engineering a mouse that shares Bea's gene variant, which could help to clarify whether the mutation revs up signalling or suppresses it.

The latest study does not define a new 'Rienhoff syndrome'. For that, Rienhoff and his collaborators would need to find other patients who share Bea's features and genetic markers. Rienhoff says that he would be relieved if he found an older person with similar symptoms who seems as vivacious as his daughter, who recently earned an orange belt in karate; it would tell him that cardiovascular complications are not pre-ordained. "If I saw a single case, I might say, 'Hallelujah', he says.

\section{Proof mooted for quantum uncertainty}

\section{BY RON COWEN}

$\mathrm{E}$ ncapsulating the strangeness of quantum mechanics is a single mathematical expression. According to every undergraduate physics textbook, the uncertainty principle states that it is impossible to simultaneously know the exact position and momentum of a subatomic particle - the more precisely one knows the particle's position at a given moment, the less precisely one can know the value of its momentum.

But the original version of the principle, put forward by physicist Werner Heisenberg in 1927, couches quantum indeterminism in a different way - as a fundamental limit to how well a detector can measure quantum properties. Heisenberg offered no direct proof for this version of his principle, and expressed his ideas "only informally and intuitively", says physicist Jos Uffink of the University of Minnesota in Minneapolis.

Now researchers say that they have a formal proof. "Our work shows that you can't measure something with an accuracy any better than the fundamental quantum uncertainty," says Paul Busch, a theoretical physicist at the University of York, UK, who with his colleagues posted the proof on 6 June on the arXiv preprint server ${ }^{1}$. Not only does the work place this measurement aspect of the uncertainty principle on solid ground - something that researchers had started to question - but it also suggests that quantum-encrypted messages can be transmitted securely.

In their theoretical work, Busch and his colleagues imagined making simultaneous measurements of a particle's position and momentum in an arbitrary quantum state. They compared the errors in such measurements to two special cases - in which either the position or the momentum of the particle is well known. They found that the combined errors in measurements of the position or momentum in these two cases obeyed Heisenberg's principle and was always smaller than for cases in which the two properties were measured simultaneously. This step allowed them to prove Heisenberg's original conjecture.

Busch and his co-authors' work "is worth being remembered and maybe even taught through textbooks as the proper version of the Heisenberg error-disturbance relation", says mathematician Hans Maassen of Radboud University in Nijmegen, the Netherlands. However, the proof has reignited a decade-long debate.

\section{POLARIZED DEBATE}

In 2003, physicist Masanao Ozawa, now at Nagoya University in Japan, proposed that the measurement-disturbance version of the Heisenberg uncertainty principle could be violated. Ozawa's theory was published in 2004 (ref. 2) and was corroborated last year, when Aephraim Steinberg and Lee Rozema at the University of Toronto, Canada, and their colleagues reported the results of an experiment in which they measured the polarization of a single photon along two perpendicular directions ${ }^{3}$.

Just like position and momentum, quantum theory predicts that the polarization along two different axes cannot simultaneously be known with certainty (see Nature http://doi. org/m27; 2012). The team adopted a strategy in which the polarization is initially probed using a series of 'weak' measurements - detections that barely disturb the system but must be repeated several times to record the same information that a single 'strong' measurement can detect. They found that, on average, the

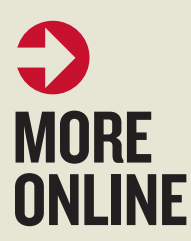

NEWS

- Online games offer trove of brain data go.nature.com/ujowtc - Violence against women at epidemic proportions go.nature. com/xtgmoh

- Blocking boozy memories reduces risk of relapse go.nature. com/wppqan
Whole human brain is mapped in 3D go.nature.com/os/b4s 